\title{
Should we use naloxone epidurally?
}

\author{
Gilbert Blaise MD
}

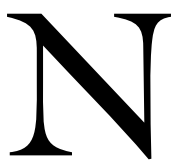

ALOXONE (6-oxy-17-propenyl morphine), a competitive antagonist of $\mathrm{Mu}$ receptors, is regularly used by anesthesiologists to reverse excessive sedation and respiratory depression following opioid administration. It is usually available on every hospital floor if epidural or intrathecal morphine is given for postoperative analgesia. Anesthesiologists treat pruritis with low doses of naloxone without reversing the analgesia induced by epidurally- or intrathecally-administered morphine. Naloxone is also used as a medication to rapidly wean patients addicted to opioids using anesthesia with full monitoring. ${ }^{1}$ Naloxone is administered by veterinarians when large, wild animals have been anesthetized with carfentanil. ${ }^{2}$ Besides these wellknown indications, naloxone has been tested in animal models and clinical trials for the possible treatment of several pathologies, including drug abuse, alcohol addiction, eating disorders, spinal cord injury, shock, cerebral and cardiac ischemia, ${ }^{3}$ and constipation induced by perimedullar morphine.

As $\mathrm{Mu}$ receptors are linked to drug addiction, $\mathrm{Mu}$ antagonists have been approved by the Food and Drug Administration to treat alcoholic patients. More recently, it has been established that morphine acting on $\mathrm{Mu}$ receptors will activate two types of $\mathrm{G}$ proteins: at micromolar concentrations, $\mathrm{Mu}$ agonists activate Gi/Go, which inhibits adenylate cyclase, decreases cyclic adenosine monophosphate (cAMP) and is responsible for the antinociceptive effect of morphine by decreasing calcium entry ${ }^{4}$ or by opening $\mathrm{K}^{+}$channel and hyperpolarizing cells $;{ }^{5}$ at pico to nanomolar concentrations, $\mathrm{Mu}$ agonists interact with Gs, which stimulates adenylate cyclase, increases cAMP, promotes $\mathrm{Ca}^{++}$influx and induces phosphoinositide hydrolysis. ${ }^{6}$ Stimulation of this pathway augments neural cell responses and is one of the mechanisms of morphine tolerance. Low doses of $\mathrm{Mu}$ antagonists such as naloxone and naltrexone preferentially block the Gs path- way, enhancing the antinociceptive action of morphine and preventing morphine tolerance; very low doses of naltrexone and probably naloxone can, by themselves, have an antinociceptive effect by potentiating endogenous endorphins. ${ }^{7}$ Another mechanism by which $\mathrm{Mu}$ receptor antagonists could potentiate the antinociceptive action of morphine is by stimulating $\mathrm{Mu}$ receptor recirculation and increasing the number of $\mathrm{Mu}$ receptors available on neural cell surfaces. ${ }^{8}$ It has been shown that naloxone can protect from brain damage induced by fentanyl and morphine $\mathrm{e}^{9,10}$ and can decrease superoxide production in microglia stimulated by Bamyloid peptide ${ }^{3}$ and inflammatory processes; ${ }^{11,12}$ this effect is stereoisomere-dependent, suggesting a possible beneficial action of naloxone in stroke and chronic cerebral pathologies such as Alzheimer's and Parkinson's disease.

Naloxone can be administered $i v$ or $s c$; after oral treatment, its bioavailability is around $2 \%$ and unpredictable. ${ }^{13}$ Are there indications for epidural naloxone? The following effects of naloxone could be considered for its peridural application: potentiation of morphine analgesia, inhibition of morphine tolerance, protection against morphine toxicity, and treatment or prevention of pruritus (as reported by Okutomi et al. in this issue of the Canadian Journal of Anesthesia), ${ }^{14}$ of respiratory depression induced by morphine, of constipation and urinary retention ${ }^{15}$ evoked by epidurallyadministered morphine.

Is naloxone safe for use as an epidural medication? In general, repeated spinal exposure to a medication for 28 days in two species at a concentration far exceeding the clinically-anticipated dose is an accepted test before proceeding clinically with epidural or spinal injection in North America. ${ }^{16}$ To the best of my knowledge, this has not been done with naloxone.

Anecdotal evidence based on limited clinical experience supports the use of epidural naloxone. Naloxone has a chemical structure that is close to

From the Department of Anesthesia, Hôpital Notre-Dame du CHUM, Montréal, Québec, Canada.

Address correspondence to: Dr. Gilbert Blaise, Laboratoire d'anesthésie, Pavillon Deschamps, porte FS-1136, Hôpital Notre-Dame du CHUM, 1560, rue Sherbrooke est, Montréal, Québec H2L 4M1, Canada. Phone: 514-890-8202; Fax: 514-412-7520;

E-mail: blaisegil@sympatico.ca 
morphine and several hundred patients have been treated with naloxone epidurally without reported toxicity. We have used a morphine-naloxone combination (ratio 1/0.08) in hundreds of patients suffering from chronic back pain: $80 \%$ had a $50 \%$ reduction of their pain scores lasting for weeks (personal unpublished observation). Despite the use of a morphinenaloxone mixture, $54 \%$ of patients complained of pruritus, but this remains lower than the $90 \%$ incidence reported when morphine is given intrathecally after a Cesarean section.

Epidural naloxone has interesting properties. It is available with and without a conservation agent and as a mixture of + and - isomeres, where + isomeres are protective against oxydative stress and - isomeres are $\mathrm{Mu}$ antagonists. Naloxone should be tested in clinical trials to establish more convincingly its safety, its role in the treatment and prevention of pruritus and its effects on analgesia. The best form (which isomeres; with or without a conservation agent) and concentration ratio of naloxone to morphine remain to be determined. The pharmacokinetics of naloxone as a single injection, compared to morphine, should also be investigated.

\section{Devrait-on utiliser la naloxone dans l'espace péridural ?}

La naloxone (6-oxy-17-propényl morphine), un antagoniste compétitif des récepteurs $\mathrm{Mu}$, est employée régulièrement par les anesthésiologistes pour renverser la sédation excessive et la dépression respiratoire qui suivent l'administration d'opioïde. Elle est habituellement disponible dans tout service hospitalier où la morphine péridurale ou intrathécale est administrée comme analgésie postopératoire. Les anesthésiologistes traitent le prurit avec de faibles doses de naloxone sans renverser l'effet analgésique induit par la morphine péridurale ou intrathécale. La naloxone est aussi utilisée avec une anesthésie sous monitorage complet ${ }^{1}$ pour sevrer rapidement les patients qui ont une dépendance aux opioïdes. La naloxone est administrée par les vétérinaires à de gros animaux sauvages anesthésiés avec du carfentanil. ${ }^{2}$ Outre ces indications bien connues, la naloxone a été étudié chez des modèles animaux et lors d'essais cli- niques comme traitement possible de certaines pathologies, y compris l'usage de drogues, l'alcoolisme, les troubles de l'alimentation, des lésions de la moelle épinière, un choc, l'ischémie cérébrale et cardiaque $^{3}$ et la constipation induite par la morphine périmédullaire.

Les récepteurs $\mathrm{Mu}$ étant liés à la toxicomanie, les antagonistes $\mathrm{Mu}$ ont été approuvés par la Food and Drug Administration américaine pour traiter les alcooliques. Plus récemment, on a démontré que la morphine agissant sur les récepteurs $\mathrm{Mu}$ active deux types de protéines $\mathrm{G}$ : à des concentrations micromolaires, les agonistes $\mathrm{Mu}$ activent les $\mathrm{Gi} / \mathrm{Go}$, lesquelles inhibent l'adénylate-cyclase, diminuent l'adénosine monophosphate cyclique (AMP cyclique) et sont responsables de l'effet antinociceptif de la morphine en réduisant l'entrée de calcium ${ }^{4}$ ou en ouvrant le canal $\mathrm{K}+$ et en hyperpolarisant les cellules $;^{5}$ pour des concentrations de pico à nanomolaires, les agonistes $\mathrm{Mu}$ interagissent avec les Gs, lesquelles stimulent l'adénylate-cyclase, augmentent l'AMP cyclique, favorisent l'influx de $\mathrm{Ca}++$ et induisent l'hydrolyse phosphoinositide. ${ }^{6}$ La stimulation de ce mécanisme augmente la réponse des cellules nerveuses et constitue l'un des mécanismes de tolérance de la morphine. De faibles doses d'antagonistes $\mathrm{Mu}$ comme la naloxone et la naltrexone bloquent de préférence le mécanisme de la protéine Gs, stimulant l'action antinociceptive de la morphine et prévenant la tolérance à la morphine ; de très faibles doses de naltrexone, et probablement de naloxone, peuvent par elles-mêmes produire un effet antinociceptif en potentialisant les endorphines endogènes. ${ }^{7}$ Un autre mécanisme par lequel les antagonistes des récepteurs $\mathrm{Mu}$ peuvent stimuler l'action antinociceptive de la morphine est la stimulation de la recirculation des récepteurs $\mathrm{Mu}$ et l'augmentation du nombre de récepteurs $\mathrm{Mu}$ disponibles à la surface des cellules nerveuses. ${ }^{8} \mathrm{On}$ a démontré que la naloxone peut protéger des dommages cérébraux induits par le fentanyl et la morphine 9,10 et qu'elle peut diminuer la production de superoxyde dans la microglie stimulée par les peptides Bamylö̈de $^{3}$ et les processus inflammatoires; ${ }^{11,12}$ cet effet est stéréoisomère-dépendant, ce qui laisse croire à une action bénéfique possible de la naloxone en cas d'accidents vasculaires cérébraux et de pathologies cérébrales chroniques comme la maladie d'Alzheimer et de Parkinson.

La naloxone peut être administrée par voie $i v$ ou $s c$; après un traitement par voie orale, sa biodisponibilité est d'environ $2 \%$ et est imprévisible. ${ }^{13} \mathrm{Y}$ a-t-il des indications pour l'administration péridurale de la naloxone ? On peut considérer les effets suivants : la 
potentialisation de l'analgésie morphinique, l'inhibition de la tolérance à la morphine, la protection contre la toxicité de la morphine et le traitement ou la prévention du prurit (comme le rapportent Okutomi et coll. dans le présent numéro du Journal), ${ }^{14}$ de la dépression respiratoire induite par la morphine, de la constipation et de la rétention urinaire ${ }^{15}$ provoquées par la morphine péridurale.

La naloxone est-elle sans danger comme médicament péridural ? En général, en Amérique du Nord, ${ }^{16}$ l'exposition rachidienne répétée à une médication pendant 28 jours, administrée à deux espèces animales, à une concentration qui dépasse de beaucoup la dose prévue en clinique est un test acceptable avant de procéder au traitement par injection péridurale ou rachidienne. À ma connaissance, cela n'a pas été fait avec la naloxone.

Des données empiriques fondées sur une expérience clinique limitée favorisent l'usage épidural de naloxone. La naloxone et la morphine ont une structure chimique semblable et des centaines de patients ont été traités avec la naloxone épidurale sans qu'on rapporte de toxicité. Nous avons utilisé une combinaison de morphine et de naloxone (ratio $1 / 0,08$ ) chez des centaines de patients souffrant de lombalgies chroniques : $80 \%$ ont noté une réduction de $50 \%$ de leur score de douleurs qui a duré des semaines (observation personnelle non publiée). Malgré l'utilisation d'un mélange de morphine et de naloxone, $54 \%$ des patients se sont plaints de prurit, mais ce pourcentage demeure plus bas que les $90 \%$ notés lors de l'administration de morphine intrathécale après une césarienne.

La naloxone péridurale possède des propriétés intéressantes. Elle est offerte avec ou sans agent conservateur et comme mélange d'isomères + et -, où les isomères + protègent contre le stress oxydatif et les isomères - sont des antagonistes $\mathrm{Mu}$. La naloxone doit être testée lors d'essais cliniques afin d'établir de façon plus convaincante son innocuité, son rôle dans le traitement et la prévention du prurit et ses effets sur l'analgésie. Les meilleurs formes (quels isomères ; avec ou sans conservateur) et ratio de concentration entre la naloxone et la morphine sont encore à déterminer. La pharmacocinétique de la naloxone en injection unique, comparée à la morphine, devrait être étudiée également.

\section{References}

1 Kienbaum P, Scherbaum N, Thurauf N, Michel MC, Gastpar M, Peters J. Acute detoxification of opioidaddicted patients with naloxone during propofol or methohexital anesthesia: a comparison of withdrawal symptoms, neuroendocrine, metabolic, and cardiovas- cular patterns. Crit Care Med 2000; 28: 969-76.

2 Moresco A, Larsen RS, Sleeman JM, Wild MA, Gaynor

$J S$. Use of naloxone to reverse carfentanil citrateinduced hypoxemia and cardiopulmonary depression in Rocky Mountain wapiti (Cervus elaphus nelsoni). J Zoo Wildl Med 2001; 32: 81-9.

3 Liu $\Upsilon$, Qin L, Wilson BC, An L, Hong JS, Liu B. Inhibition by naloxone stereoisomers of beta-amyloid peptide (1-42)-induced superoxide production in microglia and degeneration of cortical and mesencephalic neurons. J Pharmacol Exp Ther 2002; 302: 1212-9.

4 Tallent M, Dichter MA, Bell GI, Reisine T. The cloned kappa opioid receptor couples to an $\mathrm{N}$-type calcium current in undifferentiated PC-12 cells. Neuroscience 1994; 63: 1033-40.

5 Ikeda K, Kobayashi T, Ichikawa T, Usui H, Kumanishi $T$. Functional couplings of the $\delta$ - and the $\kappa$-opioid receptors with the G-protein-activated $\mathrm{K}^{+}$channel. Biochem Biophys Res Commun 1995; 208: 302-8.

6 Crain SM, Shen KF. Acute thermal hyperalgesia elicited by low-dose morphine in normal mice is blocked by ultra-low-dose naltrexone, unmasking potent opioid analgesia. Brain Res 2001; 888: 75-82.

7 Powell KJ, Abul-Husn NS, Jhamandas A, Olmstead $M C$, Beninger RJ, Jhamandas K. Paradoxical effects of the opioid antagonist naltrexone on morphine analgesia, tolerance, and reward in rats. J Pharmacol Exp Ther 2002; 300: 588-96.

8 Narita M, Mizoguchi H, Nagase H, Suzuki T, Tseng $L F$. Up-regulation of spinal $\mu$-opioid receptor function to activate G-protein by chronic naloxone treatment. Brain Res 2001; 913: 170-3.

9 Sinz EH, Kofke WA, Garman RH. Phenytoin, midazolam, and naloxone protect against fentanyl-induced brain damage in rats. Anesth Analg 2000; 91: 1443-9.

10 Kakinohana M, Marsala M, Carter C, Davison JK, Yaksh TL. Neuraxial morphine may trigger transient motor dysfunction after a noninjurious interval of spinal cord ischemia: a clinical and experimental study. Anesthesiology 2003; 98: 862-70.

11 Liu B, Du L, Kong LY, et al. Reduction by naloxone of lipopolysaccharide-induced neurotoxicity in mouse cortical neuron-glia co-cultures. Neuroscience 2000; 97 : 749-56.

12 Liu B, Jiang JW, Wilson BC, et al. Systemic infusion of naloxone reduces degeneration of rat substantia nigral dopaminergic neurons induced by intranigral injection of lipopolysaccharide. J Pharmacol Exp Ther 2000; 295: 125-32.

13 Lin M, Wittbrodt E. Low-dose oral naloxone reverses opioid-induced constipation and analgesia. J Pain Symptom Manage 2002; 23: 48-53. 
14 Okutomi T, Saito M, Mochizuki J, Amano K.

Prophylactic epidural naloxone reduces the incidence and severity of neuraxial fentanyl-induced pruritus during labour analgesia in primiparous parturients (Letter). Can J Anesth 2003; 50: 960-1.

15 Rawal N, Mollefors K, Axelsson K, Lingardh G, Widman $B$. An experimental study of urodynamic effects of epidural morphine and of naloxone reversal. Anesth Analg 1983; 62: 641-7.

16 Eisenach JC, Yaksh TL. Epidural ketamine in healthy children-what's the point? Anesth Analg 2003; 96: 626-7. 